\title{
12 Dokumentarismus als Kontrafaktik
}

Der Verbindung zwischen kontrafaktischem und dokumentarischem Erzählen hat die bisherige Forschung kaum Aufmerksamkeit gewidmet. Das Fehlen einer solchen Verbindung kann allerdings auch nur wenig überraschen, mutet doch die Begriffsverbindung ,kontrafaktischer Dokumentarismus“ vorderhand wie ein Oxymoron an. Versteht man unter „Dokumentarliteratur“ eine „mit bereits vorgefundenen, authentischen Materialien operierende Literatur“857 respektive unter „Dokumentartheater“ eine „[d]ramatische Darstellung historischer Ereignisse und Personen mit demonstrativem Authentizitätsanspruch“" ${ }^{858}$ - dies die Definitionen aus dem Reallexikon für Literaturwissenschaft -, so scheint die Option eines kontrafaktischen Dokumentarismus vorderhand auszuscheiden, zeichnet sich Kontrafaktik doch gerade durch eine Abwandlung und Variation authentischen Faktenmaterials aus. Unter den vier möglichen fiktional-realen Weltvergleichsverhältnissen - Realistik, Fantastik, Kontrafaktik und Faktik - weist der Dokumentarismus eine Affinität weniger zur Kontrafaktik als zur Faktik auf: Dokumentarisches Schreiben bedient sich einer „erkennbare[n] Referenz auf Faktisches“ und unterstellt sich damit einem „empirische[n] Wahrheitsanspruch“". 859

Die Bindung des Dokumentarismus ans Faktisch-Konkrete schließt jedoch nicht automatisch die Option eines kontrafaktischen Dokumentarismus aus. Schließlich handelt es sich auch bei der Kontrafaktik um eine Form von Literatur, welche „mit bereits vorgefundenen, authentischen Materialien“ operiert, oder, um die Terminologie der Minimaldefinition der Kontrafaktik aufzugreifen: mit realweltlichem Faktenmaterial. Nur wird dieses Material im Fall der Kontrafaktik eben nicht in authentisch-faktischer, sondern in variierter Form wiedergegeben. Tatsächlich eröffnet gerade die konstitutive Bindung dokumentarischer Texte an realweltliche Dokumente zugleich auch die Möglichkeit kontrafaktischer Realitätsreferenzen.

Wenn im Folgenden von ,Dokumentarismus ' die Rede ist, so sind damit speziell Ausprägungen eines literarischen oder fiktionalen Dokumentarismus gemeint. Derartige literarische Dokumentarismen konkurrieren in aller Regel nicht mit faktualen Dokumentarismen, prätendieren also keine strenge Faktenüberein-

857 Walter Fähnders: Dokumentarliteratur. In: Klaus Weimar (Hg.): Reallexikon der deutschen Literaturwissenschaft. Band I. Berlin 1997, S. 383-385, hier S. 383.

858 Günter Saße: Dokumentartheater. In: Klaus Weimar (Hg.): Reallexikon der deutschen Literaturwissenschaft. Band I. Berlin 1997, S. 385-388, hier S. 385.

859 Saße: Dokumentartheater, S. 386.

Ә Open Access. ( 2022 Michael Navratil, publiziert von de Gruyter. (c))BY-NC-ND Dieses Werk ist lizenziert unter einer Creative Commons Namensnennung - Nicht-kommerziell - Keine Bearbeitung 4.0 International Lizenz. https://doi.org/10.1515/9783110763119-015 
stimmung oder gar Wissenschaftlichkeit. ${ }^{860}$ Würde der Autor lediglich den Zweck der Faktenpräsentation verfolgen, so wäre es wenig einleuchtend, weshalb überhaupt auf literarische Mittel oder fiktionale Medien zurückgegriffen würde. Literarische Dokumentarismen sind in aller Regel kreative Dokumentarismen wiederum ein vordergründiges Oxymoron -, Dokumentarismen also, welche sich die Lizenzen literarischen und fiktionalen Erzählens zunutze machen und ihr spezifisches Wirkpotenzial gerade aus einer produktiven Vermittlung zwischen Faktentreue und künstlerischer Erfindung, Bearbeitung und Verfremdung gewinnen. ${ }^{861}$ Eine in den letzten Jahren intensiv diskutierte Manifestationsform eines solchen kreativen Dokumentarismus ist die sogenannte Dokufiktion. Je nach Definition können die Begriffe ,kreativer Dokumentarismus' und ,Dokufiktion“ sogar synonym zueinander verwendet werden. ${ }^{862}$

Während faktuale Dokumentarismen ein variables Verhältnis zum Bereich des Politischen unterhalten (Natur- und Tierdokumentationen beispielsweise erheben meist keinen politischen Anspruch ${ }^{863}$ ), lassen sich fiktionale Dokumentarismen in aller Regel als Formen politischen Schreibens verstehen. Der politischdidaktische Anspruch des Dokumentarismus deutet sich dabei bereits über die Etymologie des Wortes ,Dokument“ an, dass auf das lateinische Verb docere, für lehren, instruieren, unterrichten, zurückgeht. ${ }^{864}$ Als Teildefinition der Doku-

860 Vgl. Sven Hanuschek: „Ich nenne das Wahrheitsfindung“. Heinar Kipphardts Dramen und ein Konzept des Dokumentartheaters als Historiographie. Bielefeld 1993, S. 28, 69-72.

861 Klaus Harro Hilzinger weist in diesem Zusammenhang darauf hin, dass der „konstitutive Widerspruch der Form“ des Dokumentartheaters in der „Bindung ans Dokumentarische“ bei gleichzeitiger „Lösung vom Dokumentarischen“ besteht. Vgl. Klaus Harro Hilzinger: Die Dramaturgie des dokumentarischen Theaters. Tübingen 1976, S. 9.

862 Markus Wiegandt etwa versteht die Dokufiktion ,als eine Sammelbezeichnung für Texte, in denen man die Relation von Dokumentation und Fiktion als wechselseitiges Bedingungsverhältnis auffassen muss. In dieser Form gewinnt die Literatur nicht nur Anschluss an die Wirklichkeit, sondern vermittelt auch zwischen dem Vor- bzw. Weltwissen des Lesers einerseits und dem gleichwohl noch existenten ästhetischen Anspruch der Literatur als Kunst.“ (Markus Wiegandt: Chronisten der Zwischenwelten. Dokufiktion als Genre. Operationalisierung eines medienwissenschaftlichen Begriffs für die Literaturwissenschaft. Heidelberg 2017, S. 68) Eine solche Definition der Dokufiktion ist mit einem kreativen und kontrafaktischen Dokumentarismus, wie er im Folgenden diskutiert wird, bestens vereinbar.

863 Die meisten Definitionen des Politischen beziehen sich ausschließlich auf menschliche Aktanten. So lassen sich etwa Naturdokumentationen erst dadurch politisieren, dass ökologische Bedingungen als vom Handeln des Menschen - etwa dem menschengemachten Klimawandel - abhängig präsentiert werden.

864 Vgl. Werle: Fiktion und Dokument, S. 113. Die Affinität von Dokumentarischem und Politischem führt Werle unter anderem auf die große Bedeutung von Dokumenten im Kontext der Rechtsprechung zurück: „Dokumente sollen etwas zeigen oder beweisen - die damit zusammenhängende Affinität zum juristischen Kontext scheint eine Ursache dafür zu sein, dass die 
mentarliteratur gibt Nikolaus Miller an: „Dokumentarliteratur verleiht dem dokumentarischen Stoff Bedeutung und macht ihn so zum Medium einer zumeist politischen Aussage. “865 Fiktionale Dokumentarismen liefern also meistens nicht einfach ein neutrales Bild der Realität, sondern verbinden die eigene Realitäts- oder Faktenpräsentation mit normativen Wertungen und/oder einem Aufklärungsanspruch.

Fiktionstheoretisch betrachtet unterscheiden sich die Fakten des (kontrafaktischen) Dokumentarismus nicht kategorial von denjenigen des (kontrafaktischen) historischen Erzählens: Strenggenommen ist jedwedes historische Erzählen bis zu einem gewissen Grade zugleich auch dokumentarisch, da die Geschichte ja nur über Dokumente zugänglich ist; und jeder Dokumentarismus - selbst der tagesaktuelle Dokumentarismus einer Zeitung oder Nachrichtenmeldung - dokumentiert Vergangenes, also Historisches. Jenseits dieser fiktionstheoretischen Überschneidung von Dokumentarismus und historischem Erzählen lässt sich aber auch mit Blick auf die Literaturgeschichte eine enge Verbindung der beiden literarischen Formen erkennen. Besonders augenfällig ist diese Verbindung beim Dokumentardrama, dem im deutschsprachigen Raum weitaus bedeutendsten Genre dokumentarischer Literatur. ${ }^{866}$ Bereits das frühe Dokumentartheater der 1920er Jahre unter Erwin Piscator stellte nicht zuletzt eine Reaktion auf die Erfahrungen des Ersten Weltkriegs dar. ${ }^{867}$ Auch die zentralen Werke aus der Hochphase der Dokumentardramatik in den 1960er Jahren - etwa die Werke von Rolf Hochhuth, Heinar Kipphardt oder Peter Weiss ${ }^{868}$ - befassen sich sämtlich mit historischen Stoffen. ${ }^{869}$

Repräsentation von Dokumenten in der Literatur häufig auf ethische, politische und didaktische Zwecke abzielt.“ (Ebd., S. 119).

865 Nikolaus Miller: Prolegomena zu einer Poetik der Dokumentarliteratur. München 1982, S. 59.

866 Heinz Ludwig Arnold hält fest, dass sich „das Dokumentartheater [...] eher und eindrücklicher Geltung verschafft hat als die relativ selten gebliebene Reportage- und Interviewliteratur.“ (Heinz Ludwig Arnold: Vorbemerkung. In: Ders. / Stephan Reinhardt (Hg.): Dokumentarliteratur. München 1973, S. 7-12, hier S. 9) Blickt man auf die deutliche Privilegierung des Dokumentartheaters gegenüber anderen dokumentarischen Genres sowohl im Reallexikon der deutschen Literaturwissenschaft als auch im von Dieter Lamping herausgegebenen Handbuch der literarischen Gattungen, so wird man Arnolds Befund aus den frühen 1970er Jahren nach wie vor zustimmen können.

867 Vgl. Franz-Josef Deiters: „Gegossen in den Schmelztiegeln der Groß-Industrie, gehärtet und geschweißt in der Esse des Krieges“. Erwin Piscator oder Die Geburt der Theateravantgarde in den Gräben des Ersten Weltkriegs. In: Christian Klein / Franz-Josef Deiters (Hg.): Der Erste Weltkrieg in der Dramatik - deutsche und australische Perspektiven / The First World War in Drama - German and Australian Perspectives. Stuttgart 2018, S. 101-117.

868 Brian Barton identifiziert zwei „dokumentarische[...] Wellen im deutschsprachigen Theater 1924 bis 1929 und 1963 bis 1970“ (Brian Barton: Das Dokumentartheater. Stuttgart 1987, S. 1).

869 So weist etwa Sven Hanuschek auf die „weitreichenden Parallelen des Dokumentardramas mit der Historiografie“ hin (Sven Hanuschek: Dokumentardrama. In: Dieter Lamping (Hg.): 
Die große Nähe und partielle Überschneidung von historischem und dokumentarischem Erzählen erlaubt es, zahlreiche der Überlegungen zur historischen Kontrafaktik auch auf die dokumentarische Kontrafaktik zu übertragen. Entsprechend muss an dieser Stelle kein weiteres Mal auf das spannungsreiche Verhältnis von (kontrafaktischer) Faktenpräsentation und Postmoderne sowie auf die Notwendigkeit einer heuristischen Trennung zwischen fiktionalem und faktualem kontrafaktischen Erzählen eingegangen werden. Im Folgenden soll vor allem das Verhältnis der Kontrafaktik zu einigen spezifischen Genrevarianten des Dokumentarismus sowie zum Verfahren der Verfremdung erläutert werden, welches für literarische Dokumentarismen von besonderer Bedeutung ist.

Nicht alle Ausprägungen des Dokumentarismus sind mit dem Erzählverfahren der Kontrafaktik kompatibel. In einem Ausschlussverhältnis zueinander stehen Dokumentarismus und Kontrafaktik immer dann, wenn ein Text den Anspruch erhebt, seine Leserschaft erstmalig mit einem gewissen Sachverhalt vertraut zu machen, wenn der Text den Lesern also verlässliche Informationen über ein bestimmtes Ereignis liefern soll. Ein Text kann nämlich nicht neue Informationen über die reale Welt liefern und diese Informationen, die ja noch gar nicht als bekannt vorausgesetzt werden dürfen, gleichzeitig variieren. Zwar beziehen sich auch Werke der Kontrafaktik stets auf realweltliches Faktenmaterial. Doch ist dieser Faktenbezug im Falle der Kontrafaktik implizit, es ist also Aufgabe des Lesers, eine Verbindung zwischen dem explizit Ausgesagten und den implizit, in der Negation gleichsam mitgemeinten Fakten herzustellen. Die der Kontrafaktik zugrundeliegenden Fakten müssen dabei dem fiktionalen Text notwendigerweise vorgängig sein, also auch unabhängig von der Fiktion Gültigkeit beanspruchen können. Kontrafaktische Texte können - wie alle fiktionalen Texte - nicht selbst realweltliches Faktenmaterial produzieren, sondern müssen dieses Faktenmaterial als fiktionsunabhängig gültig voraussetzen. ${ }^{870}$

Entsprechend wird ein klassischer Aufklärungsdokumentarismus, welcher es zum Ziel hat, die Öffentlichkeit allererst über einen bestimmten Sachverhalt zu informieren, auf amimetische Erzählverfahren wie Kontrafaktik und Fantastik verzichten müssen. Ein bekanntes Beispiel eines solchen, am Faktisch-Authentischen orientierten Dokumentarismus sind die investigativen Reportagen Günter Wallraffs. Die Recherchemethode Wallraffs besteht darin, sich mithilfe elaborierter Verkleidungen und Masken in das Umfeld eines bestimmten Repor-

Handbuch der literarischen Gattungen. Stuttgart 2009, S. 132-136, hier S. 134). Siehe auch ders.: „Ich nenne das Wahrheitsfindung“.

870 Siehe Kapitel 4.3.3. Die reale Welt. 
tage-Ziels einzuschleusen, um anschließend seine persönlichen Eindrücke und Erfahrungen in der Rolle eines bestimmten sozialen oder ethnischen Typus einer breiten Öffentlichkeit zu präsentieren. Weite Verbreitung erlangte Wallraffs Buch Ganz unten (1985), in dem der Journalist die Ausbeutungsverhältnisse, die Ausgrenzung und den Hass beschreibt, mit denen er in der Rolle eines türkischen Gastarbeiters konfrontiert war. Wie viele dokumentarische Arbeiten verfolgen Wallraffs Reportagen eine dezidiert aufklärerische und, damit verbunden, gesellschaftskritische Agenda. Diese Agenda beruht dabei wesentlich auf dem Faktualitätsanspruch seiner Texte: Die Leser sind angehalten, zu glauben, dass Wallraff die beschriebenen Recherchen durchgeführt, die geschilderten Erfahrungen tatsächlich gemacht und sich bei der schriftlichen Darlegung um dokumentarische Präzision bemüht hat. Bestände der Verdacht auf schriftstellerische Erfindung - etwa in Form kontrafaktischer oder fantastischer Passagen -, so würde der aufklärerisch-gesellschaftskritische Impetus von Wallraffs Texten entschieden abgeschwächt. Auch in Interviewdokumentationen wie Erika Runges Bottroper Protokolle (1968) oder in historischen Materialsammlungen wie Walter Kempowskis Echolot-Projekt (1993-2005) müssen kontrafaktische oder fantastische Elemente zuverlässig ausgeschlossen werden, um den faktual-dokumentarischen und damit explizit aufklärerischen Anspruch dieser Werke aufrechterhalten zu können.

Inkompatibel ist der kontrafaktische Dokumentarismus ferner mit dem Reportagestil des New Journalism, der ab den 1960er Jahren von US-amerikanischen Autoren wie Tom Wolfe und Truman Capote entwickelt wurde. ${ }^{871}$ Die Texte des New Journalism weichen die Grenze zwischen fiktionaler Literatur und faktualem Journalismus auf, indem sie zwar einen Anspruch auf Faktentreue erheben, zugleich aber in hohem Maße auf literarische Mittel zurückgreifen, die Lizenzen fiktionalen Erzählens nutzen und die Subjektivität der Darstellung herausstellen. ${ }^{872}$ Im Rahmen des Reportageverfahrens des New Journalism sind imaginative oder subjektive Ergänzungen des jeweiligen Faktenmaterials zwar möglich und bis zu einem gewissen Grade sogar vorgesehen. Eine Erfindung jedoch, welche den Ergebnissen der Recherche auf offenkundige Weise widerspräche - wie es bei kon-

871 Siehe allgemein zum New Journalism Joan Kristin Bleicher / Bernhard Pörksen (Hg.): Grenzgänger. Formen des New Journalism. Wiesbaden 2004.

872 So wird in Truman Capotes Tatsachenroman In Cold Blood streckenweise heterodiegetisch, zugleich aber intern fokalisiert erzählt. Zipfel bemerkt zu derartigen Spezialfällen: „Diese Texte beziehen ihre Faszination gerade daraus, daß sie wissentlich und willentlich die allgemein geltenden sprachhandlungs- und erzähllogischen Grenzen zwischen faktualem und fiktional(-phantastisch)em Erzählen übertreten. Die Erzählstrukturen dieser Texte sind absichtlich paradox“ (Zipfel: Fiktion, Fiktivität, Fiktionalität, S. 169). 
trafaktischen Variationen des Faktenmaterials ja der Fall wäre -, würde dem journalistischen Anspruch, den der New Journalism ungeachtet aller literarischen Darstellungslizenzen weiterhin erhebt, zuwiderlaufen.

Durchaus vereinbar sind Kontrafaktik und Dokumentarismus hingegen dort, wo bestimmte Sachverhalte bereits als bekannt vorausgesetzt werden können, sodass die Variation dieser Sachverhalte innerhalb eines fiktionalen Werkes dann auch erkennbar ist. Die Rezeptionsstruktur wäre in diesem Fall weitgehend identisch mit derjenigen der historischen Kontrafaktik (eine strikte Unterscheidung zwischen dokumentarischen Fakten und historischen Fakten ist, wie gesagt, nicht möglich). Ein solcher kontrafaktischer Dokumentarismus liegt beispielsweise vor, wenn ein kontrafaktisches Werk als Satire eines realweltlichen Sachverhalts oder auch eines nicht-fiktionalen dokumentarischen Werkes fungiert: Man denke etwa an die zahlreichen fiktionalen Politikersatiren im Fernsehen oder im Internet, welche eine weithin bekannte charakterliche, sprachliche, habituelle etc. Eigenheit einer Person des öffentlichen Lebens dadurch kommentieren, dass sie diese Eigenheit in der künstlerischen Darstellung kontrafaktisch variieren, also über die Maßen betonen oder in auffälliger Weise aussparen. Ähnlich wie die Kontrafaktik im historischen Bereich selbst kein Wissen über den realen Geschichtsverlauf bereitstellt, so kann auch ein solcher satirisch-kontrafaktischer Dokumentarismus keine neuen Informationen über die reale Welt produzieren. ${ }^{873} \mathrm{Im}$ Gegenteil muss er eine solide Wissensbasis voraussetzen oder auf eine solche verweisen, um sein spezifisches Wirkpotenzial entfalten zu können. ${ }^{874}$ (Unberührt bleibt hiervon freilich - wie stets bei der Kontrafaktik - die Möglichkeit sekundärer epistemischer Gewinne, etwa die erkenntnisfördernde Funktion einer Satire, welche bestimmte Eigenschaften einer Person gerade in der kontrafaktischen Übertreibung besonders deutlich hervortreten lässt. ${ }^{875}$ )

873 Blume hält in diesem Kontext fest: „[Satire und Parodie] nehmen Bezug auf einen konkreten, wenn auch häufig nicht explizit benannten Gegenstand der Kritik, und sie bedürfen zu ihrer vollen Entfaltung des Erkennens dieses Gegenstands durch den Leser [...]. Da die Satire Ausdruck von Anstoßnahme ist, [...] bezieht sie sich notwendigerweise auf nichtfiktionale Konzepte innerhalb der Enzyklopädie des satirisch Schreibenden. Es ist ein abwegiger Gedanke, daß ein Satiriker den Gegenstand, den er kritisch ins Visier nimmt, eigens vollständig erfindet - jede Kritik liefe dann von vornherein ins Leere.“ (Blume: Fiktion und Weltwissen, S. 207).

874 Beispielsweise bedienen sich die satirisch-fiktionalen Artikel der Website Der Postillon regelmäBig kontrafaktischer Referenzstrukturen. Deren erfolgreiche Aktualisierung wird unter anderem dadurch sichergestellt, dass die Website zahlreiche Links zu faktualen Zeitungsmeldungen enthält, sodass die reale Faktenbasis einer bestimmten satirisch-kontrafaktischen Darstellung mühelos überprüft werden kann. Vgl. Der Postillon. Quelle: https://www.der-postillon.com/ (Zugriff: 27.07.2021).

875 So bemerkt Köppe: „Das generelle Verbot unmittelbarer Schlüsse vom Gehalt fiktionaler Sätze auf die Wirklichkeit ist sowohl mit, sekundären` Informationsabsichten von Autoren als 
Bei einem Text jedoch, der sich gar nicht mehr um eine inhaltlich adäquate Präsentation des Quellen- oder Recherchematerials bemüht, würde sich die Frage stellen, ob der Begriff ,Dokumentarismus“ hier überhaupt noch sinnvoll anwendbar ist. „Wenn die Dokumente ihre Funktion richtig erfüllen und konkrete, unwiderlegbare Beziehungen zur Außenwelt herstellen sollen“, so konstatiert Brian Barton, „müssen sie auch nach ihrer Bearbeitung [...] in einem gewissen Sinne ,authentisch“ bleiben. “876 Für einen Dokumentarismus, der sich massiv vom den ihm zugrundeliegenden Dokumenten entfernt, ergeben sich entsprechend eine Reihe von Problemen: Eine inhaltliche Abweichung von wenig bekanntem dokumentarischen Material könnte etwa zur Folge haben, dass die Abweichung gar nicht erkannt wird. Auch droht durch allzu große Verfremdung der Dokumente ein etwaiger politisch-kritischer Anspruch verlorenzugehen, wie er vom Gros literarischer Dokumentarismen erhoben wird und wesentlich auf einer erkennbaren Nähe zum Quellenmaterial beruht. ${ }^{877}$ Weicht ein Text demgegenüber von allgemein bekanntem Faktenmaterial ab, so würde er von den meisten Lesern wohl eher dem Genrebereich der Alternativgeschichte als demjenigen des Dokumentarismus zugeschlagen, da es wenig sinnvoll erscheint, gut bekannte (historische) Fakten ein weiteres Mal zu ,dokumentieren' - und sei es selbst im variierenden Modus der Kontrafaktik. Angesichts dieser konzeptionellen Schwierigkeiten überrascht es nicht, dass sich kaum Beispiele für einen Dokumentarismus anführen lassen, der das ihm zugrundeliegende Material auf erkennbare und signifikante Weise inhaltlich verändert.

Die inhaltliche Veränderung bietet jedoch nur eine Realisierungsmöglichkeit der Kontrafaktik. Kontrafaktische Variationen können sich nämlich durchaus auch auf die formale Dimension des Faktenmaterials beziehen: Der Schwerpunkt einer kontrafaktischen Variation muss nicht notwendigerweise auf inhaltlichpropositionale Aspekte gelegt werden, sondern kann auch die Auswahl, sinnliche Präsentation oder Verfremdung des Materials betreffen (wobei freilich stets $\mathrm{zu}$ berücksichtigen ist, dass die Trennung von Form und Inhalt sich nicht

auch mit ,sekundären“ leserseitigen Interpretationen vereinbar, in denen Bezüge zur Wirklichkeit hergestellt werden. [...] [Diese] Möglichkeit spricht in jedem Fall dafür, dass keine ,Durchbrechung‘ oder ,Verletzung der Fiktionalitätsinstitution (bzw. der diese Institution konstituierenden Regeln) vorliegen muss, wenn ein fiktionaler Text zur Vermittlung von Einsichten eingesetzt und/oder verwendet wird.“ (Köppe: Die Institution Fiktionalität, S. 47).

876 Barton: Das Dokumentartheater, S. 3.

877 Zum spannungsreichen Verhältnis von Dokument, Verfremdung und politischem Anspruch bemerkt Hilzinger: „Wenn [...] der kritische Gehalt mit dem Grad der Verfremdung steigt, liegt doch andererseits die Bedeutung und Wirkung des dokumentarischen Theaters mit in der Unmittelbarkeit der Fakten begründet“ (Hilzinger: Die Dramaturgie des dokumentarischen Theaters, S. 9). Siehe auch Barton: Das Dokumentartheater, S. 4-6. 
konsequent durchhalten lässt und dass formale Variationen ab einem gewissen Grade der Intensität notwendigerweise in inhaltliche Variationen umschlagen und vice versa). Für das Genre des Dokumentarismus ist eine solche Option der formalen Kontrafaktik von besonderem Interesse, bildet die Frage nach Möglichkeiten und Grenzen einer formalen Veränderung von Faktenmaterial doch seit jeher eine Grundfrage der Genrediskussion rund um den literarischen Dokumentarismus. Peter Weiss etwa schreibt in seinen Notizen zum dokumentarischen Theater: „Das dokumentarische Theater enthält sich jeder Erfindung, es übernimmt authentisches Material und gibt dies, im Inhalt unverändert, in der Form bearbeitet, von der Bühne aus wieder.“878 Die Möglichkeit der formalen Bearbeitung wird von Weiß für den Dokumentarismus also explizit eingeräumt. Weitgehend ungeklärt bleibt in Weiss' Definition hingegen die Frage, welche genauen Aspekte diese formale Bearbeitung umfasst und wie weit sie gehen kann, damit der „Inhalt“ weiterhin als „unverändert“ gelten kann. Tatsächlich unterscheiden sich verschiedene Formen des Dokumentarismus sehr deutlich in Art und Umfang ihrer Bearbeitung des Recherchematerials. ${ }^{879}$ Peter Weiss selbst etwa greift für sein Stück Die Ermittlung (1965) auf die Protokolle Bernd Naumanns zum Frankfurter Auschwitz-Prozess zurück. So gut wie alle Aussagen der Richter, Verteidiger und Angeklagten in Die Ermittlung lassen sich auf die Protokolle Naumanns zurückführen. Allerdings nimmt Weiss dabei einige signifikante Veränderungen am Textmaterial vor. Das Wort ,Jude، etwa, das in den Protokollen vielfach vorkommt, wird in Die Ermittlung ausgespart. ${ }^{880}$ Rolf Hochhuth hingegen stellt in den umfänglichen Nebentexten seines Stücks Der Stellvertreter (1963) die Tatsächlichkeit des Geschehens heraus, nämlich das Versäumnis von Papst Pius XII., öffentlich Position zur Deportation und massenhaften Vernichtung der Juden während des Zweiten Weltkriegs zu beziehen. ${ }^{881}$ In der „theatralischen Präsentation“ jedoch, so fasst Günter Saße zusammen, ,bedient sich Hochhuth ganz tradi-

878 Peter Weiss: Notizen zum dokumentarischen Theater. In: Joachim Fiebach (Hg.): Manifeste europäischen Theaters. Grotowski bis Schleef. Berlin 2003, S. 67-73, hier S. 67 f.

879 Vgl. Barton: Das Dokumentartheater, S. 2.

880 Vgl. Jesko Bender: Dekonstruktiver Dokumentarismus. Peter Weiss' Ermittlung und die Möglichkeiten literarischer Repräsentation von Realität im Schatten von Auschwitz. In: Kultur \& Gespenster 3 (2007), S. 168-185, hier S. 169, 173 f.

881 Hochhuth fügt der Druckfassung seines Stücks einen „historischen Anhang“ hinzu, an dessen Beginn er betont: „Die folgenden Anmerkungen zu umstrittenen Geschehnissen und Aussagen sollen [...] beweisen, daß der Verfasser des Dramas sich die freie Entfaltung der Phantasie nur so weit erlaubt hat, als es nötig war, um das vorliegende historische Rohmaterial überhaupt zu einem Bühnenstück gestalten zu können. Die Wirklichkeit blieb stets respektiert, sie wurde aber entschlackt.“ (Rolf Hochhuth: Der Stellvertreter. Ein christliches Trauerspiel. Reinbek bei Hamburg ${ }^{37} 2004$, S. 381). 
tioneller Mittel: erfundene Begebenheiten, erfundene Personen, erfundene Dialoge arrangiert er nach dem überkommenen Muster einer Entscheidungsdramaturgie, in der Protagonisten und Antagonisten sich gegenüberstehen. “882 Milo Rau schließlich lässt in seinem Reenactment Breiviks Erklärung (2012) eine deutsche Übersetzung der Rede des norwegischen Massenmörders Anders Behring Breivik vor dem Osloer Amtsgericht weitgehend wortgetreu vortragen. Allerdings wird die Rede des Rechtsterroristen Breivik von einer Schauspielerin mit türkischem Migrationshintergrund vorgetragen, die ein Barack-Obama-Fan-T-Shirt trägt und demonstrativ Kaugummi kaut, sodass der rechts-ideologische Inhalt von Breiviks Erklärung performativ unterlaufen wird. ${ }^{883}$ In allen drei Fällen handelt es sich um künstlerisch-dokumentarische Arbeiten, die nicht so sehr den Inhalt der zugrundeliegenden Dokumente variieren, sondern eher auf formale Mittel der Verfremdung und Faktenvariation zurückgreifen. ${ }^{884}$ Je nachdem, wie weit diese formale Variation geht - und vor allem: wie relevant diese Variation als Variation für die Interpretation des jeweiligen Werkes ist -, kann sie durchaus als eine Manifestation formaler Kontrafaktik angesehen werden. ${ }^{885}$

Es scheint dies der geeignete Ort für einige grundlegende Bemerkungen zum Zusammenhang von Kontrafaktik und Verfremdung zu sein. Die Verfremdung als künstlerisches Verfahren bietet die Möglichkeit einer - meist formalen - Variation des Materials, die sich gleichwohl niemals vollständig vom Ausgangsmate-

882 Günter Saße: Faktizität und Fiktionalität. Literaturtheoretische Überlegungen am Beispiel des Dokumentartheaters. In: Wirkendes Wort 36/1 (1986), S. 15-26, hier S. 19.

883 Vgl. Robert Walter-Jochum: (Ent-)Schärfungen. Terrorideologien als Material von Reenactments bei Romuald Karmakar und Milo Rau. In: Stefan Neuhaus / Immanuel Nover (Hg.): Das Politische in der Literatur der Gegenwart. Berlin / New York 2019, S. 255-272, hier S. 268.

884 Eine detaillierte Untersuchung der genannten Werke würde freilich zwischen den verschiedenen Formen und Funktionen der Bezugnahme auf realweltliches Faktenmaterial zu unterscheiden haben. Rolf Hochhuth versucht mit Der Stellvertreter letztlich vor allem Wissenslücken hinsichtlich der realen historischen Ereignisse zu schließen, indem er historische Tatsachen in einem fiktionalen Medium vermittelt (vgl. Saße: Faktizität und Fiktionalität, S. 19). Peter Weiss und Milo Rau hingegen erzeugen in ihren Werken keine direkte Referenz auf die jeweiligen historischen Ereignisse - Auschwitz respektive die Anschläge in Norwegen am 22. Juli 2011 -, sondern beziehen sich auf faktuale Texte, die im Kontext dieser Ereignisse entstanden sind: im Falle von Die Ermittlung also auf den Frankfurter Auschwitz-Prozess, im Falle von Breiviks Erklärung auf die Gerichtsrede des Terroristen. Indem Weiss und Rau also sprachliche Äußerungen in - unter anderem - sprachlichen Medien dokumentieren, vermeiden sie erstens einen für den Dokumentarismus stets problematischen Medienwechsel und entgehen zweitens dem Vorwurf, anhand einer künstlerischen Repräsentation zum ,realen Kern` von Genozid und Massenmord vordringen zu wollen.

885 Siehe zur formalen Kontrafaktik im bildkünstlerischen Bereich Kapitel 4.3.4. Das Faktenverständnis der Kontrafaktik. 
rial entfernt: Verfremdung ist immer Verfremdung von etwas. Beziehen sich nun Verfremdungsverfahren innerhalb fiktionaler Medien auf realweltliches Faktenmaterial - was keineswegs immer der Fall ist -, so können sich durchaus kontrafaktische Referenzstrukturen ergeben. Zwar variieren kontrafaktische Werke nicht notwendigerweise (und de facto eher selten) formale Aspekte des jeweiligen Faktenmaterials; auch beziehen sich verfremdende Verfahren nicht zwingend auf die formale Dimension der Darstellung (wenngleich formale Verfremdungen der Regelfall sein dürften). Betrachtet man allerdings Fälle formaler Kontrafaktik gemeinsam mit Fällen faktenbezogener (formaler) Verfremdung, so wird man eine bedeutende Schnittmenge zwischen beiden Feldern beobachten können.

Die Verfremdung als eine mögliche Realisationsform der Kontrafaktik ist freilich nicht auf den Genrebereich des Dokumentarismus beschränkt, sondern kann theoretisch in allen Genres zum Einsatz kommen, die mit der Kontrafaktik kompatibel sind. Allerdings ist der Rückgriff auf Techniken der Verfremdung im Fall des kontrafaktischen Dokumentarismus besonders naheliegend, bietet die Verfremdung doch die Möglichkeit, einen bestimmten Informationsgehalt inhaltlich unverändert zu lassen - und somit den Bezug zu den vorgängigen Dokumenten, über welche der Dokumentarismus sich ja allererst definiert, beizubehalten -, zugleich aber eine künstlerische Bearbeitung, formale Abwandlung oder eben kontrafaktische Variation des Faktenmaterials vorzunehmen. ${ }^{886}$

Das genaue wechselseitige Verhältnis von Kontrafaktik und Verfremdung lässt sich nun theoretisch nicht problemlos bestimmen. Dies hat vor allem darin seinen Grund, dass es sich bei der Kontrafaktik um einen fiktionstheoretisch präzise definierbaren Begriff handelt, während der Begriffsgehalt des Wortes ,Verfremdung' historischen und kontextuellen Schwankungen unterworfen ist. Je nach Anwendungsbereich oszilliert er etwa zwischen wahrnehmungstheoretischen, formal-technischen und ideologischen Deutungen. Ursprünglich geht der Begriff ,Verfremdung (russisch: ostranenie) auf den russischen Formalismus zu Beginn des 20. Jahrhunderts zurück. In seinem klassischen Aufsatz Die Kunst als Verfahren aus dem Jahre 1916 definiert Viktor Šklovskij das „Verfahren der Kunst [als] das Verfahren der ,Verfremdung“ der Dinge und das Verfahren der erschwerten Form, ein Verfahren, das die Schwierigkeit und Länge der Wahrnehmung steigert““ ${ }^{887}$ Šklovskij sieht in der Kunst ein Mittel, um „,aus dem Automatismus

886 Siehe hierzu auch das Kapitel „Illusion und Verfremdung“ in Hilzinger: Die Dramaturgie des dokumentarischen Theaters, S. 12-64.

887 Viktor Šklovskij: Die Kunst als Verfahren. In: Jurij Striedter (Hg.): Russischer Formalismus. Texte zur allgemeinen Literaturtheorie und zur Theorie der Prosa. München 1971, S. 4-35, hier S. 15. 
der Wahrnehmung herausgelöst [zu] werden“. ${ }^{888}$ Die Möglichkeit poetischer Schöpfung - also etwa die Integration fantastischer oder kontrafaktischer Elemente in eine erzählte Welt - berücksichtigt Šklovskij in seiner primär wahrnehmungstheoretisch orientierten Lesart der Verfremdung allerdings nicht.

Von weitreichender Bedeutung für die deutschsprachige Verwendung des Begriffs ,Verfremdung' ist bekanntlich seine Aneignung und Modifikation durch Bertolt Brecht im Rahmen der Theorie des Epischen Theaters. ${ }^{889} \mathrm{Im}$ Text Über experimentelles Theater definiert Brecht die Verfremdung wie folgt: „Einen Vorgang oder einen Charakter verfremden heißt zunächst einfach, dem Vorgang oder dem Charakter das Selbstverständliche, Bekannte, Einleuchtende zu nehmen und über ihn Staunen und Neugierde zu erzeugen.“890 Hinsichtlich der Ent-Automatisierung der Wahrnehmung besteht bei Brecht also Kontinuität mit der Theorie Šklovskijs. Bezüglich der technischen Realisierungsformen gehen die von Brecht vorgesehenen Verfremdungstechniken jedoch deutlich über die von Šklovskij angeführten, ungewohnten Formen der Beschreibung hinaus: Die Verfremdungseffekte - oder schlicht V-Effekte - in Brechts Stücken umfassen narrative Passagen, Songs, Texteinblendungen auf der Bühne, lautes Lesen von Regieanweisungen, satirische und parabelhafte Figurendarstellungen und vieles mehr.

Zusätzlich zu ihrer formalen Dimension wird die Verfremdung bei Brecht mit einem kritisch-politischen Impetus versehen. Verfremden heißt für Brecht „Historisieren, heißt Vorgänge und Personen als historisch, also als vergänglich darstellen. ${ }^{\text {" } 91}$ Letztlich besteht der Zweck der Verfremdung für Brecht darin, die gesellschaftlichen Zustände als veränderbar darzustellen und damit idealerweise ihre reale Veränderung vorzubereiten. ${ }^{892}$ Diese politische Aufladung des Verfremdungskonzepts hat traditionsbildend gewirkt: Die meisten literarischen Texte, die seit Brecht auf Verfremdungsverfahren zurückgreifen - darunter auch das Gros literarisch-dokumentarischer Texte bis in die Gegenwart -, nutzen

$\mathbf{8 8 8}$ Šklovskij: Die Kunst als Verfahren, S. 15.

889 Einen detaillierten Vergleich zwischen Šklovskijs und Brechts Konzeptionen der Verfremdung bietet Hans Günther: Verfremdung: Brecht und Šklovskij. In: Susi K. Frank u. a. (Hg.): Gedächtnis und Phantasma. Festschrift für Renate Lachmann. München 2001, S. 137-145.

890 Bertolt Brecht: Über experimentelles Theater. In: Ders.: Werke. Große kommentierte Berliner und Frankfurter Ausgabe (= GBA). Hg. v. Werner Hecht u. a. Frankfurt a. M. 1988ff., Bd. 22: Schriften 2, S. 540-557, hier S. 554.

891 Brecht: Über experimentelles Theater, S. $554 \mathrm{f}$.

892 Günther bemerkt hierzu: „Geht es Šklovskij um die Wiederherstellung des ästhetischen Empfindens durch Erschwerung der Wahrnehmung, so interessiert Brecht über das AuffälligMachen hinaus die Möglichkeit der intellektuellen Intervention des Zuschauers, das ,Dazwischenkommen“ des Urteils.“ (Günther: Verfremdung: Brecht und Šklovskij, S. 141). 
die Verfremdung zu politisch-kritischen Zwecken. ${ }^{893}$ Eine Assoziation von Verfremdung, politischem Schreiben und (formaler) Kontrafaktik, wie sie in der vorliegenden Arbeit vorgeschlagen wird, erscheint insofern besonders naheliegend.

Verfremdung kann, so konstatiert Hans Günther, „auf allen Ebenen des literarischen Werks ansetzen“ und „sehr unterschiedliche Funktionen haben.“894 Nicht alle Spielarten der Verfremdung lassen sich dabei plausibel mit dem Bereich der Kontrafaktik in Verbindung bringen. Beispielsweise müssen Verfremdungsverfahren nicht notwendigerweise auf konkrete Fakten referieren, sondern können etwa auch auf allgemeine Realitätsannahmen Bezug nehmen: Wenn etwa in Brechts Stück Der gute Mensch von Sezuan die Identifikation mit der Hauptfigur, der gutmütigen Prostituierten Shen Te, dadurch erschwert wird, dass ein anderer, egoistisch-pragmatischer Teil von Shen Tes Persönlichkeit in Form des gerissenen Geschäftsmanns Shui Ta eigenständig auf der Bühne agiert, so liegt hier keine kontrafaktische Referenz auf realweltliches Faktenmaterial vor. Vielmehr bezieht sich dieser Verfremdungseffekt - wie in den meisten Stücken Brechts - auf allgemeine Realitätsannahmen im Sinne der Realistik, etwa auf die Schwierigkeiten, in einer ökonomisch prekären Situation moralisch richtig zu handeln. Auch modifizieren Verfremdungseffekte nicht notwendigerweise die fiktionale Welt selbst, sondern mitunter lediglich den Wahrnehmungsprozess derselben. (Die von Šklovskij angeführten Beispiele ungewohnter Beschreibungsformen im Werk Tolstois mögen zwar Gewohnheiten der Wahrnehmung ,entautomatisieren'; den Gegenstand der Wahrnehmung verändern sie aber nicht. ${ }^{895}$ ) Und schlussendlich zielen manche Formen der Verfremdung eher auf eine komplette Zerstörung der fiktionalen Welt als auf eine Variation derselben. So versteht sich das Epische Theater Klaus-Detlef Müller zufolge „nicht als Wirklichkeit, sondern verweist explizit auf seinen Schein- und Spielcharakter (DESILLUSIONIERUNG), um auf die Wirklichkeit hinzuweisen." ${ }^{896}$ Wenn etwa Schauspieler im Theater aus ihren Rollen fallen, um sich mit Personen im Publikum zu unterhalten, so liegt hier ein Fall von Anti-Illusionismus oder Fiktions-Störung vor, der mit der Zwei-Welten-Struktur der Kontrafaktik - dem konstitutiven Vergleich eines realen Elements mit der Variation desselben Elements innerhalb

893 Vgl. Hans Günther: Verfremdung 2 . In: Jan-Dirk Müller (Hg.): Reallexikon der deutschen Literaturwissenschaft. Bd. III. Berlin 2003, S. 753-755, hier S. 753.

894 Günther: Verfremdung, S. 754.

895 Programmatisch formuliert Šklovskij: „[D]ie Kunst ist ein Mittel, das Machen einer Sache zu erleben; das Gemachte hingegen ist in der Kunst unwichtig. “(Šklovskij: Die Kunst als Verfahren, S. 15).

896 Klaus-Detlef Müller: Episches Theater. In: Klaus Weimar (Hg.): Reallexikon der deutschen Literaturwissenschaft. Bd. I. Berlin 1997, S. 468-471, hier S. 468. 
einer fiktionalen Welt - nicht mehr vereinbar ist. Ein Extrembeispiel in dieser Hinsicht bilden Inszenierungen des postdramatischen Theaters, die mitunter gar keine fiktionale Welt mehr entstehen lassen, sondern sich auf die Wiedergabe (nicht-narrativen) Textmaterials oder Akte ,reiner‘, präsentischer Performanz beschränken.

Konvergenzen von Kontrafaktik und Verfremdung ergeben sich hingegen dort, wo Formen der Verfremdung realweltliche Fakten oder faktuale Dokumente zum Ausgangsmaterial nehmen und dieses Material auf hermeneutisch signifikante Weise variieren. Innerhalb von Brechts Euvre wären hier etwa die Stücke mit historischem oder dokumentarischem Hintergrund zu nennen: So lässt sich die titelgebende Hauptfigur des Stückes Der aufhaltsame Aufstieg des Arturo Ui durchaus als satirisch-kontrafaktische Variante von Adolf Hitler, möglicherweise sogar von Al Capone interpretieren ${ }^{897}$ (wobei man sich hier wiederum eher im Bereich der Alternativgeschichte als im Bereich des kontrafaktischen Dokumentariums bewegt).

Von besonderer Bedeutung für postmodern-avantgardistische Formen des künstlerischen Dokumentarismus ist die Möglichkeit einer formalen Verfremdung, die sich primär auf dokumentarisches Sprachmaterial bezieht. Die dokumentarischen (respektive meta-dokumentarischen) Arbeiten Elfriede Jelineks etwa setzen in ihrem künstlerischen Bearbeitungsprozess häufig an der sprachlichen Form selbst an, indem sie Sprecherrollen veruneindeutigen, Texte rhythmisieren, collagieren oder mit Fremdmaterial anreichern. Freilich etablieren sprachliche Auffälligkeiten in künstlerischen Texten nicht automatisch kontrafaktische Referenzstrukturen, sondern können auch andere, etwa poetische, Funktionen erfüllen. Wenn allerdings in einem fiktionalen Werk Sprachmaterial eingefügt wird, das einerseits auf Recherchen oder realweltlichen Dokumenten beruht und andererseits in einer solchen Weise präsentiert wird, dass faktische Referenzen auszuschließen sind - etwa einfach deshalb, weil ein realer Mensch so nicht sprechen würde -, dann können sich durchaus kontrafaktische Referenzstrukturen ergeben.

Formal-verfremdende Dokumentarismen fungieren in der Gegenwart häufig als Manifestationen eines kritischen Meta-Dokumentarismus. Solche kreativen Dokumentarismen dokumentieren nicht mehr selbst, sondern liefern anhand vielfältiger Verfremdungsverfahren künstlerisch-kritische Kommentare zu vorgängigen Dokumentarismen; sie bilden also gewissermaßen Dokumentarismen zweiter Stufe, im Verhältnis nämlich zu Dokumentarismen erster Stufe, die ihrerseits durchaus den

897 Vgl. Raimund Gerz: Der Aufstieg des Arturo Ui. In: Jan Knopf (Hg.): Brecht-Handbuch in fünf Bänden. Bd. 1: Stücke. Stuttgart / Weimar 2001, S. 459-474, hier S. 461. 
Anspruch erheben, konkretes Weltwissen zu präsentieren. ${ }^{898}$ Derartige kritische Meta-Dokumentarismen, wie sie sich in der deutschsprachigen Gegenwartsliteratur etwa bei Alexander Kluge oder Elfriede Jelinek finden, behalten einen deutlichen Bezug zum dokumentarischen Quellenmaterial bei, ohne deshalb jedoch die Möglichkeiten künstlerischer Gestaltungsprozesse - inklusive denjenigen der Kontrafaktik - zu beschränken. Anstatt die Kunst dem Primat des Dokumentarisch-Faktischen unterzuordnen, wird hier umgekehrt der Dokumentarismus mit Mitteln der Kunst reflektiert und dabei einer kritischen Prüfung unterzogen.

Die dokumentarischen Arbeiten Kathrin Rögglas, welche im folgenden Kapitel im Zentrum stehen sollen, schließen an beide beschriebenen Tendenzen an: In ihren dokumentarischen Texten nutzt Röggla sowohl die Möglichkeit sprachlich-formaler Verfremdung von Recherchematerial als auch die Wirkungspotenziale eines kritischen Meta-Dokumentarismus. Sprachbearbeitung und (Sprach-) Kritik stehen dabei in Rögglas Werk in einem engen Abhängigkeitsverhältnis zueinander. Im Folgenden wird zu diskutieren sein, auf welche Weise sich Rögglas kreativer Dokumentarismus kontrafaktischer Referenzstrukturen bedient und inwiefern der Rückgriff auf eine vor allem formale Kontrafaktik zum kritisch-politischen Projekt von Rögglas Schreiben beiträgt.

898 So zeigt etwa Aline Vennemann, dass Elfriede Jelinek und Peter Wagner in ihren Dramen zwar auf die dokumentarische Tradition Bezug nehme, dabei aber keine strikt dokumentarische Methode anwenden, sondern vielmehr den Dokumentarismus selbst zum Thema machen. Vgl. Aline Vennemann: Zwischen Postdramatik und Postdokumentarismus. Peter Wagner, Elfriede Jelinek und das Dokumentartheater. In: Germanica 54 (2014), S. 25-37, hier S. 34. 